\title{
Spinal Interneurons Differentiate Sequentially from Those Driving the Fastest Swimming Movements in Larval Zebrafish to Those Driving the Slowest Ones
}

\author{
David L. McLean and Joseph R. Fetcho \\ Department of Neurobiology and Behavior, Cornell University, Ithaca, New York 14853
}

\begin{abstract}
Studies of neuronal networks have revealed few general principles that link patterns of development with later functional roles. While investigating the neural control of movements, we recently discovered a topographic map in the spinal cord of larval zebrafish that relates the position of motoneurons and interneurons to their order of recruitment during swimming. Here, we show that the map reflects an orderly pattern of differentiation of neurons driving different movements. First, we use high-speed filming to show that large-amplitude swimming movements with bending along much of the body appear first, with smaller, regional swimming movements emerging later. Next, using whole-cell patch recordings, we demonstrate that the excitatory circuits that drive large-amplitude, fast swimming movements at larval stages are present and functional early on in embryos. Finally, we systematically assess the orderly emergence of spinal circuits according to swimming speed using transgenic fish expressing the photoconvertible protein Kaede to track neuronal differentiation in vivo. We conclude that a simple principle governs the development of spinal networks in which the neurons driving the fastest, most powerful swimming in larvae develop first with ones that drive increasingly weaker and slower larval movements layered on over time. Because the neurons are arranged by time of differentiation in the spinal cord, the result is a topographic map that represents the speed/strength of movements at which neurons are recruited and the temporal emergence of networks. This pattern may represent a general feature of neuronal network development throughout the brain and spinal cord.
\end{abstract}

\section{Introduction}

The ability to move relies, in part, on networks of rhythmically active neurons in the spinal cord, the so-called central pattern generator (CPG) for locomotion. Historically, research has focused on the components of the spinal CPG and their ability to generate stable locomotor rhythms (for review, see Roberts et al., 1998; Stein et al., 1998; Falgairolle et al., 2006; Grillner, 2006; Kiehn, 2006; Goulding, 2009). Much less is known about those features of spinal networks that allow movement at many different speeds and strengths (Li et al., 2007a; Berkowitz, 2008; Liao and Fetcho, 2008; Crone et al., 2009).

We recently described a functional pattern of organization in the spinal cord of larval zebrafish that underlies their ability to swim over a range of speeds (McLean et al., 2007). Motoneurons and excitatory interneurons closest to the ventral edge of the spinal cord are active at slow swimming speeds, with progressively more dorsal cells recruited as larvae speed up. In support of the functional relevance of this topographic

\footnotetext{
Received July 9, 2009; revised Sept. 3, 2009; accepted Sept. 11, 2009.

This work was supported by a fellowship (NS44728, D.L.M.) and a grant (NS26539, J.R.F.) from the National Institutes of Health. We are extremely grateful to Shin-ichi Higashijima for providing us with the alx:GFP, alx:Kaede, and $H u C$ :Kaede transgenic fish. We also thank Lindsay Heller for fish care and members of the laboratory for numerous discussions and comments on this manuscript.

Correspondence should be addressed to Joe Fetcho at the above address. E-mail: jrf49@cornell.edu.

D. L. McLean's present address: Department of Neurobiology and Physiology, Northwestern University, Evanston, IL 60208 .

DOI:10.1523/JNEUROSCI.3277-09.2009

Copyright $\odot 2009$ Society for Neuroscience $\quad$ 0270-6474/09/2913566-12\$15.00/0
}

map of recruitment, ablation of only the ventral-most excitatory interneurons perturbs slow swimming movements (McLean et al., 2007).

In zebrafish, the dorsal-most motoneurons and excitatory interneurons that participate in the most powerful movements are also the earliest born (Myers et al., 1986; Westerfield et al., 1986; Liu and Westerfield, 1988; Beattie et al., 1997; Kimura et al., 2006). This suggests that the regular dorsoventral pattern of recruitment we observed might be a reflection of developmental order, with neurons participating in faster larval circuits emerging first and occupying more dorsal positions, and progressively slower circuits being added ventrally as the animal develops. While the evidence from earliest born neurons is consistent with this idea, the relationships between the emergence of motor behavior, the timing of neuronal differentiation, and the later topographic map of speed have not been explored.

Here, we examine the development of motor activity and behavior in zebrafish and relate these to the temporal order of differentiation of neurons in the map. Our data show that large-amplitude movements and the neurons that later drive fast larval movements emerge first during development, with a gradual layering on of slower larval networks and behaviors with age. Because the position of the neurons in the spinal cord reflects their time of differentiation, the result is an orderly map of movement speed in larvae that also represents the temporal emergence of the circuits. An examination of the literature in light of this work reveals evidence suggesting that this might reflect a broad pattern of circuit development among vertebrates, including humans. 


\section{Materials and Methods}

Fish care. All experiments were performed on zebrafish (Danio rerio) between 1 and $5 \mathrm{~d}$ of age obtained from a laboratory stock of wild-type and transgenic adults. Embryos at $2 \mathrm{~d}$ of age were selected while still in their egg casing [between 48 and $54 \mathrm{~h}$ postfertilization (hpf)]. Larvae selected at $4 \mathrm{~d}$ of age were spontaneously swimming (between 96 and 106 hpf). Embryos and larvae were raised at $28.5^{\circ} \mathrm{C}$ in the same system as adults (Aquatic Ecosystems), but experiments were performed at room temperature $\left(\sim 22-26^{\circ} \mathrm{C}\right)$. At these early ages, embryonic and larval fish are still nourished by the remnants of their yolk sac. All procedures conform to the National Institutes of Health guidelines regarding animal experimentation and were approved by Cornell University's Institutional Animal Care and Use Committee.

High-speed kinematics. The very fast swimming movements of embryos and larvae were captured at 1000 frames per second using a highspeed imaging system (Redlake). Fish were placed in Hank's solution within a circular dish, lined with agarose. The agarose lining provided a low-contrast boundary for filming. Embryos can swim for periods of seconds, which would normally carry them out of the field of view. With the low-contrast agarose lining, we could keep the embryos in the field of view and capture the slower movements that occur near the end of the swimming episode. Episodes of swimming were elicited by a tactile stimulus near the tail. Analysis of the degree of yaw was performed automatically using custom-written software in LabView (National Instruments). Two regions of the body were selected near the head (5-15\% body length) and tail (85-95\% body length) and the absolute value for yaw per half cycle was measured for each for the duration of the episode. For purposes of comparison, half cycle values in milliseconds were converted to swimming frequency in $\mathrm{Hz}(1000 / n \times 2)$. Selections along the midline were based on thresholded images that maximized the detection of the whole fish and were consistent between trials. For analysis, 5 swimming episodes were measured for each embryo (all evoked, because they rarely swim without a stimulus) and 10 swimming episodes were measured for each larva ( 5 evoked, 5 spontaneous).

Electrophysiological recordings. Whole-cell patch and peripheral motor nerve recordings were performed similarly to those described previously (Drapeau et al., 1999; Masino and Fetcho, 2005). Larvae were first anesthetized in $0.02 \%$ tricaine methanesulphonate (w/v MS-222; Western Chemical International) in Hank's solution and then briefly treated with $\alpha$-bungarotoxin (Sigma-Aldrich) dissolved in extracellular solution (1 $\mathrm{mg} / \mathrm{ml}$, for composition see below). After successful immobilization (5-10 $\mathrm{min})$, larvae were transferred to a Sylgard-coated, glass-bottomed dish containing extracellular solution (ionic composition in $\mathrm{mmol} \cdot \mathrm{L}^{-1}$ : $134 \mathrm{NaCl}, 2.9 \mathrm{KCl}, 1.2 \mathrm{MgCl}_{2}, 10 \mathrm{HEPES}, 10$ glucose, $2.1 \mathrm{CaCl}_{2}$; adjusted to $\mathrm{pH} 7.8$ with $\mathrm{NaOH}$ ) and secured to the bottom using custom-etched tungsten pins placed through the notocord. For recordings of motoneuron activity from peripheral nerves, the skin was removed from the ear to just past the anus to expose the intermyotomal clefts. To record from spinal cells and motor nerves, muscle overlying one segment was removed manually to expose the spinal cord. After the dissection, the dish containing the preparation was transferred to the physiological recording apparatus, in which extracellular solution was constantly perfused at room temperature.

Micropipettes were created from thin walled, filamented glass capillaries (A-M Systems) pulled on a Flaming/Brown puller (Sutter Instrument). For patch recordings from spinal cells, micropipettes were pulled to tip diameters of $\sim 2-4 \mu \mathrm{m}$, yielding $10-20 \mathrm{M} \Omega$ resistances. For extracellular recordings of peripheral nerves, these same micropipettes were broken to make tip diameters of $\sim 20-50 \mu \mathrm{m}$ and polished using a microforge (model MF-830, Narishige International USA). Patch electrodes were first backfilled with intracellular solution (ionic composition in $\mathrm{mmol} \cdot \mathrm{L}^{-1}$ : $125 \mathrm{~K}$-gluconate, $2.5 \mathrm{MgCl}_{2}, 10 \mathrm{EGTA}, 10 \mathrm{HEPES}, 4$ $\mathrm{Na}_{2} \mathrm{ATP}$; adjusted to $\mathrm{pH} 7.3$ with $\mathrm{KOH}$ ) and then advanced into the exposed portion of spinal cord using a motorized micromanipulator (model MP-225, Sutter Instrument), while maintaining positive pressure $(\sim 25-50 \mathrm{mmHg}$ ) via a pneumatic transducer (model DPM-1B, Bio-Tek Instruments) to avoid tip clogging. When the tip of the micropipette was in close proximity to the soma of the targeted cell, a gigaohm seal was obtained by either equilibrating the micropipette to atmosphere or applying gentle suction. A holding current was then applied to maintain the cell at $-65 \mathrm{mV}$ once the micropipette had become cell attached. At this point, we would assess the firing activity of the cells during swimming to compare with recordings obtained in whole-cell mode. Whole-cell recordings were obtained by penetrating the membrane using gentle suction pulses. Standard corrections for bridge balance and capacitance were then applied. Cells were typically targeted within the first or second cell layer. For simultaneous motor nerve recordings, nerve micropipettes were filled with extracellular solution and lowered onto the intermyotomal clefts, and gentle suction was then applied via a pneumatic transducer to obtain a tight seal.

Whole-cell and peripheral nerve recordings were acquired using a MultiClamp 700A amplifier (Molecular Devices), a Digidata series 1322A digitizer (Molecular Devices), and pClamp 8.2 software (Molecular Devices). Electrical signals from spinal cells were filtered at $30 \mathrm{kHz}$ and digitized at $63 \mathrm{kHz}$ at a gain of $20\left(R_{\mathrm{f}}=5 \mathrm{G} \Omega\right)$. Only cells that had stable membrane potentials at or below $-40 \mathrm{mV}$ were included in this study. A junction potential using this recording solution has already been calculated at $16 \mathrm{mV}$, which would result in a shift of measured potentials $16 \mathrm{mV}$ in the negative direction (Higashijima et al., 2004b). Since this junction potential would not greatly impact on our conclusions we did not correct for it. Extracellular signals from peripheral nerves were recorded in current-clamp mode at a gain of 1000, with the low- and high-frequency cutoffs set at 100 and $4000 \mathrm{~Hz}$, respectively.

We included a fluorescent dye in the patch solution $(0.025 \%$ sulforhodamine-B acid chloride, Sigma-Aldrich) so we could assess the morphology of each neuron immediately after the recording. Fluorescent images were acquired with a CCD camera (C-72-CCD; Dage MTI) and a frame grabber (LG3; Scion) controlled by NIH ImageJ software. Images of recorded cells were graphically reconstructed using Abobe Illustrator (Adobe Systems).

For analysis of swimming in embryos, we measured five swimming episodes evoked by a brief electrical stimulus ( $<1 \mathrm{~ms}$ in duration $1-10 \mathrm{~V})$ delivered via a tungsten electrode placed near the tail (DS2 Isolated Stimulator, Digitimer, Ltd., Welwyn Garden City, England). For analysis of swimming in larvae, we measured a minimum of five evoked swimming episodes per fish. In addition, we measured swimming in larvae generated spontaneously and in response to a flashlight during a $30-60 \mathrm{~s}$ period, which contained a variable number of swimming episodes. Our dataset for larvae includes data collected during the course of previous studies (McLean et al., 2007, 2008). Specifically, this includes the 10 larvae used for fictive motor burst comparisons, 10 of the 15 primary motoneurons, all 30 secondary motoneurons, 25 of the 36 circumferential descending ( $\mathrm{CiD}$ ) cells (herein referred to as "CiDs") and 12 of the 15 dorsally displaced CiDs. The features used for comparison here were not quantified in the prior reports.

For measurements of spike number versus swimming frequency, we used the spikes themselves as a frequency measure, rather than nerve root activity. This way we could use a thresholding function in the automated analysis software to pick out the number of spikes per cycle and the frequency of that cycle, as determined by the time between the first spikes in consecutive cycles. This made the analysis easier. For the most part, spiking activity faithfully reflected nerve activity, although there was some variability. This variability was not an impediment, however, because the purpose of this study was to compare spiking behavior at different stages and we were doing so using the same method of analysis at the different stages. All data were analyzed off-line using DataView (software by William Heitler, University of St Andrews, St Andrews, Scotland; http://www.st-andrews.ac.uk/ wjh/dataview/).

For analysis of position versus minimum firing frequency, size, and input resistance, the dorsoventral location was determined by averaging three measurements from the bottom of spinal cord to the middle of the cell body by using either ImageJ or Zeiss LSM 510 software. This value was then normalized to an average of three measurements of the total dorsoventral extent of spinal cord. Recruitment using patch physiology was determined by averaging the three slowest swimming frequencies from the total number of cycles in five swimming episodes in which a neuron fired at least one action potential in consecutive cycles. Because 
we used spikes as a measure of swimming frequency, we could not include in our analysis the occasions when cells fired in the preceding cycle but failed to do so in the following one. Soma cross-sectional area was determined by averaging three measurements from differential interference contrast images. Resistance measurements were calculated from an average of five hyperpolarizing square current pulses between 20 and 50 $\mathrm{pA}$, in a linear current-voltage range for all the neurons tested. Location measurements were always performed first, so they would not be influenced by frequency measurements, which were less subjective.

In vivo imaging. Photoconversion and imaging of transgenic fish were performed using a confocal microscope (Zeiss LSM 510, Carl Zeiss). For photoconversion experiments, fish were anesthetized in MS-222, placed in a glass-bottomed dish (WillCo-dish, WillCo Wells), and illuminated for 1-2 min using a DAPI epifluorescence filter set. Conversion from green to red was confirmed using FITC and rhodamine epifluorescence settings. Photoconverted fish were then kept at $28.5^{\circ} \mathrm{C}$ in a light-tight container until the day of imaging.

Targeted electroporations and retrograde filling of multipolar commissural descending (MCoD) cells (herein referred to as "MCoDs") with fluorescent dyes were performed as described previously (Hale et al., 2001; Ritter et al., 2001; Bhatt et al., 2004). Briefly, for dye electroporations, fish were anesthetized and then embedded in a glass-bottomed dish left side up in $1.4 \%$ low-melting-point agarose (Sigma-Aldrich) made up in Hank's solution. Using an electrophysiology setup, a patch micropipette filled with $10 \%$ tetramethylrhodamine (3000 molecular weight, Invitrogen) diluted in patch solution was then carefully advanced through the skin and muscle into spinal cord until the tip was in direct contact with the soma of the MCoD. A brief electrical stimulus was then applied (1-5 V, $1 \mathrm{~s}$ train of 1-ms-duration pulses at $100 \mathrm{~Hz})$ via a silver wire within the pipette attached to an isolated pulse stimulator (model 2100 , A-M Systems). Successful filling of the cell was quickly confirmed by switching to an epifluorescent light source. The larva was then carefully removed from the agarose and allowed to recover for at least $4-6 \mathrm{~h}$ in Hank's solution containing $1 \%$ of the antibiotic penicillin streptomycin (Sigma-Aldrich).

For backfilling MCoD cells, fish were anesthetized and placed on an agar plate. Solution containing anesthetic was gently wicked away and then a glass micropipette was carefully advanced into caudal spinal cord $(\sim 10$ segments from the end of the tail) using a manual micromanipulator (Narishige International USA). A solution containing 20\% AlexaFluor-647 (10,000 molecular weight, Invitrogen) in Hank's was then pressure injected into ventral spinal cord using a picospritzer (General Valve). Larvae were allowed to recover for at least 4-6 h in Hank's solution containing $1 \%$ penicillin-streptomycin. Labeled arrays of MCoDs were confirmed using an epifluorescence microscope (Leica MZ 16FA, Leica Microsystems). Analysis of cell position was performed as described above using Zeiss software.

Statistical analysis. Comparisons of means were done in JMP (SAS Institute) by ANOVA either with $t$ tests or with a Tukey-Kramer Honestly Significant Difference test to give a conservative estimate of differences when there were multiple comparisons. Tests for significant nonzero correlations between different measures were tested in $\mathrm{Mi}$ crosoft Excel. $p<0.05$ was set as the level of a significant difference.

\section{Results}

Developmental changes in swimming movements

Our first goal was to determine how swimming movements in embryos compared with those produced in larvae, as this might provide some clues to potential developmental differences. From 27 hpf onward, zebrafish embryos removed from the egg will swim in response to tactile stimuli (Saint-Amant and Drapeau, 1998; Downes and Granato, 2006). The embryos can swim for several seconds, which very often carries them out of the field of view of the high-speed camera. They also have a tendency to stop swimming abruptly when they encounter an obstacle (e.g., the side of the petri dish). To more completely characterize the movements throughout a full swimming episode, we filmed embryos in a dish containing an outer ring of agar. This served two

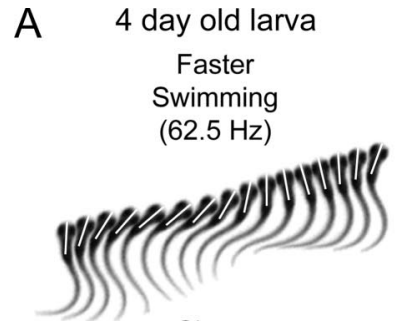

Slower
Swimming

$(25 \mathrm{~Hz})$

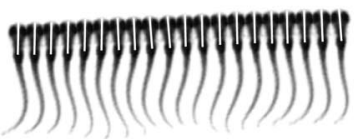

C

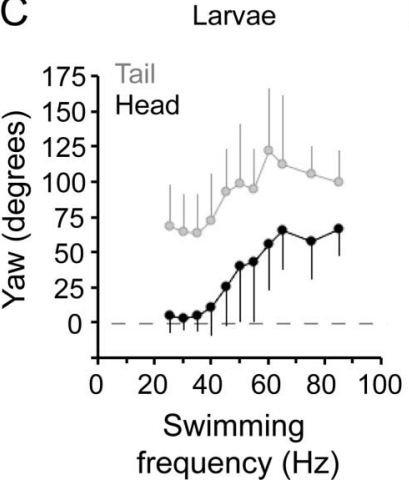

B

\section{2 day old embryo}

Faster

Swimming

$(58.8 \mathrm{~Hz})$

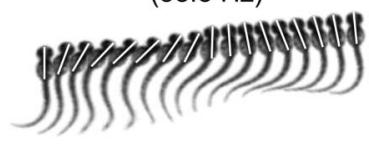

Slower

Swimming

$(25 \mathrm{~Hz})$

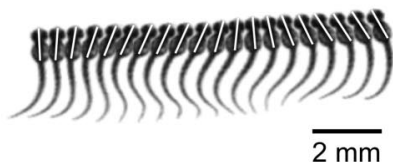

$\mathrm{D}$

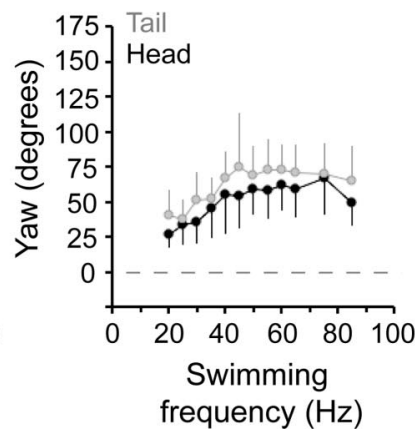

Figure 1. $\quad A, B$, Kinematic analysis of swimming movements in zebrafish embryos and larvae. $A$, Successive, overlapping images captured at 1000 frames per second of a larval zebrafish swimming at $\sim 60 \mathrm{~Hz}$ (faster swimming, top) and $25 \mathrm{~Hz}$ (slower swimming, bottom). Top images are illustrated every millisecond, and at the bottom they are every $2 \mathrm{~ms}$. B, Successive, overlapping images of an embryonic zebrafish swimming around $60 \mathrm{~Hz}$ (faster swimming, top) and around $25 \mathrm{~Hz}$ (slower swimming, bottom). As in $\boldsymbol{A}$, top images are illustrated every millisecond, and at the bottom they are every $2 \mathrm{~ms}$. White bars in $\boldsymbol{A}$ and $\boldsymbol{B}$ illustrate the lateral displacement, or yaw, of the head during swimming. $(-\boldsymbol{D}$, Plots of larval ( $(\boldsymbol{C})$ and embryonic (D) head (black circles) and tail (gray circles) yaw during various speeds of swimming. Data are binned and averaged every $5 \mathrm{~Hz}$. Errors are SDs. These data are compiled from 25 episodes of stimulus-induced swimming from five embryos and 50 episodes of swimming (25 spontaneous, 25 stimulus-induced) from five larvae.

purposes; first, it kept the embryo within the field of view so the entire swimming episode was available to be filmed and, second, the softer contact surface prevented a premature termination of the episode.

We began by filming posthatching 4-d-old (96-120 hpf) zebrafish larvae in this environment. Prior work indicated that there might be two discrete slow and fast modes of swimming in the larvae (Budick and O'Malley, 2000; Buss and Drapeau, 2001; Müller and van Leeuwen, 2004; Thorsen et al., 2004). Our own more recent work using automated analysis of trunk movements suggests that these modes actually represent two ends of a continuum and that zebrafish larvae can smoothly grade from one into the other as they speed up or slow down (McLean et al., 2008). In the agar-lined arena we observed the same pattern, which is illustrated in Figure 1. In zebrafish larvae, the fastest swimming speeds involve bending all along the body with lateral movements of the head and tail (Fig. $1 A$, top) and at slower speeds these movements become restricted to the tail, with little or no head movement (Fig. $1 A$, bottom).

In embryos removed from their eggs just before hatching $(2 \mathrm{~d}$ old or 48-72 hpf), we were able to film swimming at the begin- 
Table 1. Developmental comparison of neuronal properties, motor patterns, and behavior

\begin{tabular}{|c|c|c|c|c|c|c|}
\hline & \multicolumn{3}{|c|}{ 2-d-old embryos } & \multicolumn{3}{|c|}{ 4-d-old larvae } \\
\hline & Mean & SD & $n$ & Mean & SD & $n$ \\
\hline \multicolumn{7}{|l|}{ Head yaw (degrees) } \\
\hline$<40 \mathrm{~Hz}$ & 46.1 & 6.7 & 5 & $5.7^{*}$ & 1.9 & 5 \\
\hline$>40 \mathrm{~Hz}$ & 59.5 & 3.4 & 5 & 53.4 & 3.4 & 5 \\
\hline \multicolumn{7}{|l|}{ Tail yaw (degrees) } \\
\hline$<40 \mathrm{~Hz}$ & 59.2 & 8.0 & 5 & 65.7 & 5.9 & 5 \\
\hline$>40 \mathrm{~Hz}$ & 74.4 & 11.1 & 5 & $107.4^{*}$ & 6.9 & 5 \\
\hline \multicolumn{7}{|l|}{ Motor burst durations (ms) } \\
\hline$<40 \mathrm{~Hz}$ & 3.5 & 1.3 & 10 & $11.2^{*}$ & 1.7 & 10 \\
\hline$>40 \mathrm{~Hz}$ & 3.1 & 1.0 & 10 & 4.8 & 0.8 & 10 \\
\hline \multicolumn{7}{|l|}{ Spike number (per cycle) } \\
\hline Primary motoneurons & $1.23^{*}$ & 0.19 & 15 & $2.72^{*}$ & 0.84 & 15 \\
\hline Secondary motoneurons & $1.12^{*}$ & 0.19 & 30 & $3.09^{*}$ & 1.83 & 30 \\
\hline Rhythmic CiDs & $1.21^{*}$ & 0.17 & 20 & $2.76^{*}$ & 1.00 & 36 \\
\hline \multicolumn{7}{|l|}{ D-V position (normalized) } \\
\hline Primary motoneurons & $0.48^{*}$ & 0.04 & 15 & $0.56^{*}$ & 0.04 & 15 \\
\hline Displaced CiDs & $0.68^{*}$ & 0.05 & 15 & $0.75^{*}$ & 0.05 & 15 \\
\hline \multicolumn{7}{|l|}{ Soma size $\left(\mu \mathrm{m}^{2}\right)$} \\
\hline Primary motoneurons & $51.1^{*}$ & 6.1 & 15 & $76.5^{*}$ & 17.8 & 15 \\
\hline Displaced CiDs & $32.9^{*}$ & 4.2 & 15 & $26.2^{*}$ & 4.2 & 15 \\
\hline \multicolumn{7}{|l|}{ Input resistance ( $\mathrm{M} \Omega$ ) } \\
\hline Primary motoneurons & $267.3^{*}$ & 94.1 & 15 & $160.3^{*}$ & 69.2 & 15 \\
\hline Displaced CiDs & 621.1 & 120.3 & 15 & 708.5 & 159.2 & 15 \\
\hline
\end{tabular}

Data are reported as the mean, the SD, and the number $(n)$ of animals. Asterisks for yaw data indicate values that are significantly different from all other means for the same body region (head or tail). The others in the same category showed no differences from one another. Similarly, the asterisk for motor burst duration is the only value that was significantly different from all of the other durations. Asterisks for other measures indicate a significant difference between embryonic and larval values for that cell type. Data for 4-d-old larvae analyzed and tabulated here include data originally collected for other work (McLean et al., 2007, 2008). See Materials and Methods for details. D-V, Dorsoventral.

ning or the end of an episode and observed a similar range of frequencies to larvae, from 20 up to $85 \mathrm{~Hz}$. While the movements at the highest frequencies were comparable to those observed in larvae, with substantial bending all along the body (Fig. $1 \mathrm{~B}$, top), the movements at lower frequencies were strikingly different from low-frequency larval movements. Instead of the tailrestricted movements produced at low frequencies in larvae, lowfrequency bends in embryos still displayed a considerable amount of head yaw, with bending all along the body (Fig. $1 B$, bottom).

This difference is more clearly illustrated by comparing data quantifying head and tail yaw as a function of swimming frequency, as in Figure 1, $C$ and $D$. The yaw clearly decreased as swimming frequency declined in both larvae (Fig. $1 C$ ) and embryos (Fig. $1 D$ ). In larvae, however, head yaw decreased more quickly. In fact, below $40 \mathrm{~Hz}$, a frequency typical of spontaneous, slower larval swimming (Thorsen et al., 2004), larvae produced significantly less head yaw than embryos (Table 1 ), on average $<6$ degrees compared with just over 45 for embryos. We also noticed that larvae generated significantly more tail yaw than embryos (Table 1).

Thus, although embryos can swim over a range of frequencies like the larvae, the low-frequency movements in embryos involve displacements of the body more like the movements produced by larvae when they are swimming quickly. This is consistent with the possibility that the circuitry driving high-frequency movements in free-swimming larvae emerges early, but operates over a broader range of swimming frequencies in embryos. The distinctive movements produced later by larvae when they are moving slowly are also consistent with the possible involvement of different, perhaps additional, circuitry that produces movements at the low end of the frequency and speed range in free-swimming animals.

\section{A 2 day old embryo}

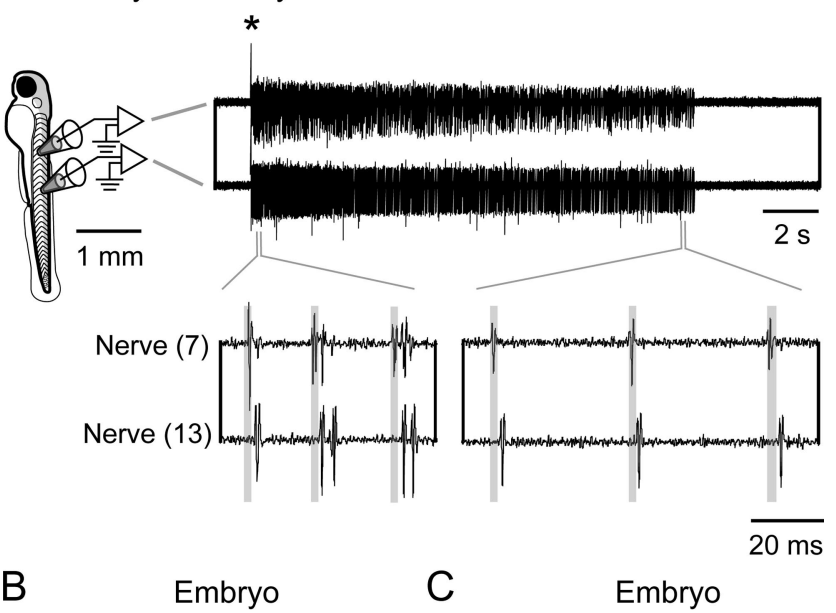

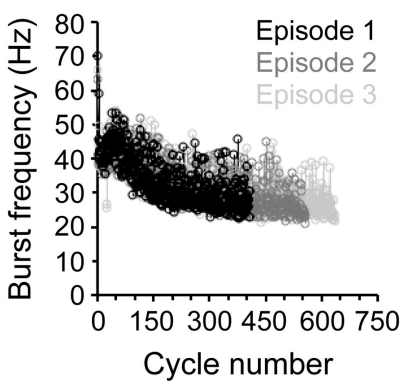

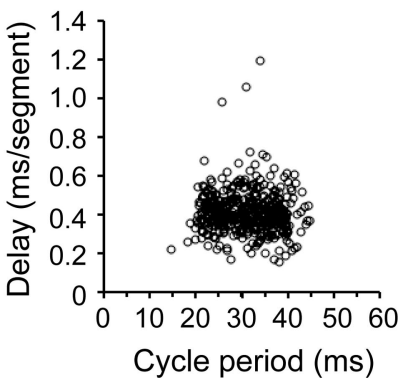

Figure 2. Fictive swimming motor pattern in embryos. $\boldsymbol{A}$, Schematic on the left illustrates the position from which motor nerve recordings illustrated on the right are taken (7th and 13th body segments). An electrical stimulus delivered to the tail was used to elicit swimming (artifact at asterisk). Top right, Single episode of swimming on a slower time base; bottom right, illustration, on a faster time base, of motor bursts that would drive cyclical bending. Transparent gray boxes illustrate the head-to-tail delay of motor bursts. $\boldsymbol{B}$, Plot of motor nerve burst frequency (measured from the tail) with respect to the cycle in the episode from three electrically evoked episodes in the same embryo. C, Plot of head-tail motor burst delay versus cycle period in a single embryo. These data are compiled from one episode of electrical stimulus-induced swimming from one embryo. Correlation values: $r=-0.08, p=0.10, n=406$ cycles.

\section{The swimming motor pattern in prehatching embryos}

The preceding analysis of swimming movements showed that embryos just before hatching can swim over the same frequency range as larvae, albeit using movements that more closely resemble fast larval swimming. We next asked whether there are differences in the motor pattern underlying embryonic versus larval swimming? If so, this might reflect differences in the underlying networks. To investigate this possibility, we recorded from the peripheral motor nerves of paralyzed embryos and examined the fictive motor activity over the full range of swimming frequencies. Examples of the set-up and recordings obtained can be seen in Figure 2. In embryos, a brief electrical stimulus elicited a single episode of swimming that could last several seconds (Fig. $2 \mathrm{~A}$, top). Immediately after the stimulus, swimming frequency was highest and had a tendency to decrease during the course of the episode (Fig. $2 \mathrm{~A}$, bottom). This progressive decline in swimming frequency has been quantified for three swimming episodes in Figure $2 \mathrm{~B}$. A decline in swimming frequency is also observed following a brief electrical stimulus in larvae (McLean et al., 2008), although fictive swimming in embryos lasts much longer, as does their real swimming behavior.

Within the swimming episodes, motor bursts in embryos were relatively brief, averaging just below $4 \mathrm{~ms}$ in duration (Table 1). A quantitative analysis showed that motor burst durations between 
embryos and larvae were only significant at frequencies of swimming $<40 \mathrm{~Hz}$ (Table 1). This is because the short bursts over the entire frequency range in embryos are more like those at higher frequencies in larvae and very different from the longer duration bursts evident at low swimming frequencies in larvae (Masino and Fetcho, 2005; McLean et al., 2008). Another major difference between embryos and larvae was related to the delay of motor bursts along the body. In embryos, intersegmental delays did not change in a consistent way with swimming frequency (Fig. $2 \mathrm{~A}$, bottom, gray-shaded boxes, and $C$ ). During swimming in adult fish, delays scale with cycle period, such that the longer the cycle period (lower frequency) the longer the delay along the body (Grillner and Kashin, 1976). We know this relationship is also present in zebrafish larvae (Masino and Fetcho, 2005; McLean et al., 2008). Quantification of these relationships in five embryos showed no consistent relationship between delay and cycle period. Specifically, of the 25 episodes measured, 8 episodes were positively correlated, 13 episodes were negatively correlated, and 4 showed no significant correlation. Figure $2 C$ shows an example from one embryo that demonstrates the lack of correlation between the delay and cycle period (see corresponding figure legends for statistical data cited here and throughout the text).

Together, these electrophysiological recordings are consistent with the behavioral analysis, in that the embryonic swimming motor pattern does not change substantially throughout its frequency range and more closely resembles high-frequency larval swimming with its shorter motor burst durations. The fact that the larval pattern differs from embryos most noticeably at lower frequencies and demonstrates proper scaling of intersegmental delays could be explained by changes in spinal circuitry during development. We next investigated how motoneurons and excitatory interneurons were organized to produce swimming activity in embryos to reveal any differences between embryos and larvae that might underlie the developmental differences in behavior.

\section{Firing patterns of motoneurons during fictive swimming in prehatching embryos}

In zebrafish, motoneurons are divided into primary and secondary classes based on their respective emergence during development (Myers et al., 1986; Westerfield et al., 1986). The secondary motoneurons, which develop later, are located ventral to primaries. Our previous work has described the pattern of recruitment for motoneurons in larvae, in which the ventral-most secondary motoneurons are active at the slowest swimming frequencies, with more dorsal secondaries and eventually primaries recruited as larvae swim at higher and higher frequencies (McLean et al., 2007). We examined whether this pattern was also evident during embryonic swimming.

Figure 3 illustrates recordings from the two classes of motoneurons in 2-d-old embryos, combined with nerve recordings to monitor motor output. When we recorded from primary motoneurons in embryonic zebrafish, we found they did not fire at the slowest frequencies, but were recruited as frequency increased (Fig. $3 A$ ). This is similar to the activity of primary motoneurons in larvae, which also are recruited at higher frequencies than secondaries (McLean et al., 2007). The motoneuron types did, however, differ in the extent of firing per cycle. Embryonic primaries fired on average one or two spikes per cycle, while in larvae they fired over twice that number-a difference that proved statistically significant after analysis (Table 1). In Figure 3, $B$ and $C$, we have illustrated recordings from secondary motoneurons at different dorsoventral locations in

\section{A 2 day old primary motoneuron}

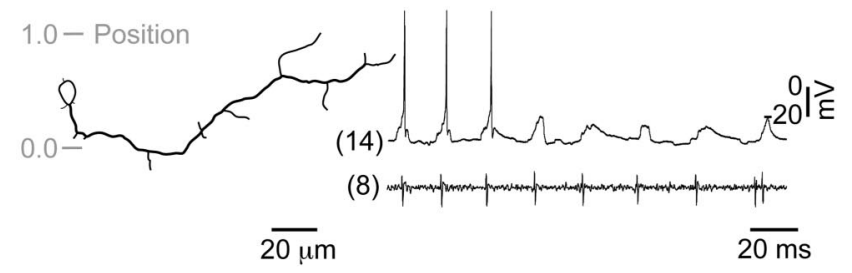

B 2 day old secondary motoneuron (dorsal)

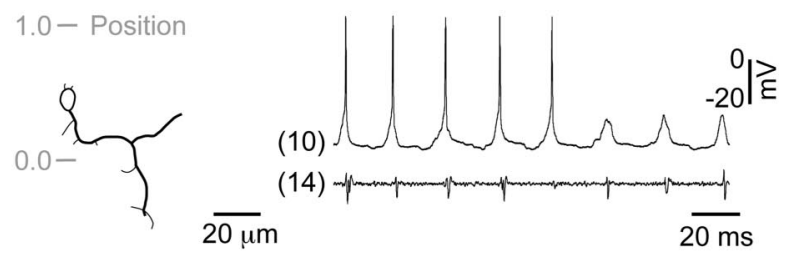

C 2 day old secondary motoneuron (ventral)
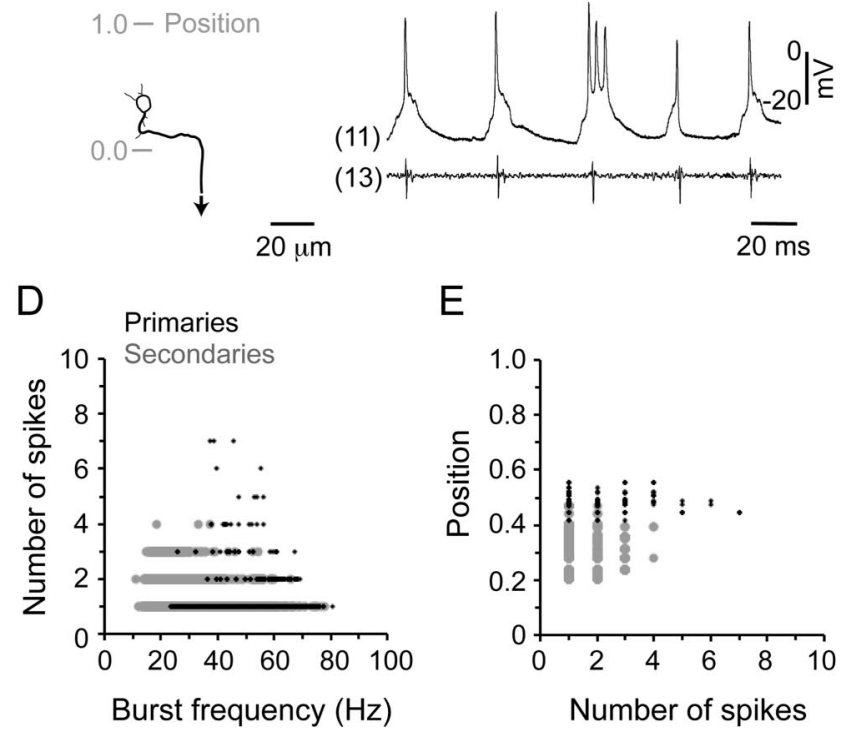

E

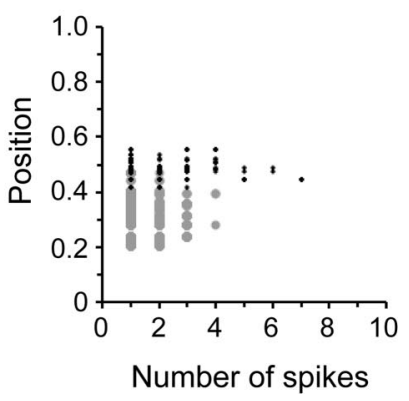

Figure 3. Motoneuron firing during fictive swimming. $\boldsymbol{A}$, Recording from a primary motoneuron in an embryo, with a reconstructed drawing of the cell on the left and the corresponding physiology immediately to the right. Gray-shaded lines indicate the position with respect to the dorsal (1.0) and ventral (0.0) edges of spinal cord. The respective segment from which the cell and the nerve recording were obtained is indicated in parentheses. $\boldsymbol{B}$, Recording from a dorsally located secondary motoneuron is organized as detailed in $\boldsymbol{A}$. $\boldsymbol{C}$, Recording from a ventrally located secondary motoneuron is organized as detailed in $\boldsymbol{A}$. An arrow indicates continuation of the axon out of the field of view. D, Plot of the number of spikes per cycle generated by motoneurons versus swimming frequency. Data represent 11,167 cycles from 30 secondary motoneurons (gray circles) and 1123 cycles from 15 primaries (black dots). $\boldsymbol{E}$, Plot of the somatic dorsoventral position of motoneurons versus the number of spikes. Graphs are plotted from the same dataset in $\boldsymbol{D}$.

embryos, which demonstrate a similar pattern of ventral to dorsal recruitment as larval secondaries. Like primary motoneurons, among secondaries there was a significant increase in the extent of firing per cycle in larvae (Table 1).

We then took a closer look at the relationship between spiking activity and swimming frequency in embryos. In larvae, a greater variability in spike number per cycle occurs at relatively lower swimming frequencies, with progressively fewer spikes occurring more reliably as they swim faster (McLean et al., 2008). In embryonic secondary motoneurons, this also appeared to be the case (Fig. 3D), with a greater variability in spike number occurring at relatively lower frequencies. In embryonic primary motoneu- 

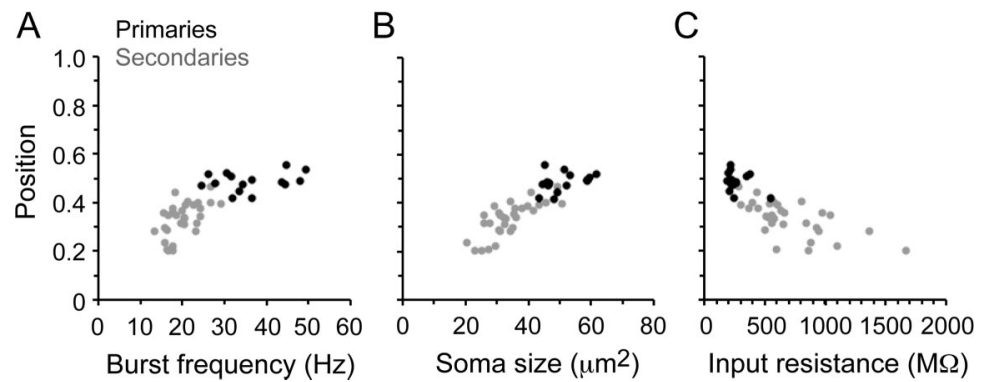

Figure 4. Motoneuron recruitment, size, and excitability in embryos. $A$, Plot of spinal cord position versus swimming frequency for 15 primary motoneuron somata (black circles) and 30 secondary motoneurons (gray circles). Correlation values: $r=0.28, p=$ $0.30, n=15$ (primaries), $r=0.56, p<0.01, n=30$ (secondaries), $r=0.77, p<0.0001, n=45$ (combined primaries and secondaries). $\boldsymbol{B}$, Plot of soma position versus soma size for the same motoneurons in $\boldsymbol{A}$. Correlation values: $r=0.36, p=0.19, n=$ 15 (primaries), $r=0.76, p<0.0001, n=30$ (secondaries), $r=0.87, p<0.0001, n=45$ (combined). C, Plot of soma position versus input resistance for the same motoneurons in $\boldsymbol{A}$ and $\boldsymbol{B}$. Correlation values: $r=-0.32, p=0.25, n=15$ (primaries), $r=$ $-0.64, p<0.001, n=30$ (secondaries), $r=-0.79, p<0.0001, n=45$ (combined). Each data point in $A-C$ represents the mean value for each cell.

rons, the broadest range of spike number occurred at the middle frequencies over which they were active (Fig. 3D). When we plotted the number of spikes against the position of the motoneurons, as in Figure 3E, there was no clear dorsoventral gradient in the ability of motoneurons to spike multiply (although some primaries could fire more spikes than secondaries).

These data demonstrate a developmental change in the firing of motoneurons, with increased spiking per cycle in larvae. The data also show that at both stages the most variability is evident at mid- to low swimming frequencies. These findings are consistent with the motor patterns recorded during development.

\section{Measuring the recruitment, size,}

\section{and input resistance of embryonic motoneurons}

Our previous work described correlations between the position of motoneurons, their size and their input resistance in larvae, which also correlate with their recruitment patterns during swimming (McLean et al., 2007). Recordings from individual embryonic motoneurons, as illustrated in Figure 3, suggested that they might show the ventral to dorsal recruitment pattern we have described in larvae. In Figure 4, we have plotted position versus minimum frequency of recruitment, size, and input resistance of the 45 embryonic motoneurons, as we did in prior studies of larvae.

As in larvae, when primary and secondary motoneuron data were pooled there were significant relationships between dorsoventral position and the minimum frequency of activation during swimming (Fig. 4A), soma size (Fig. 4B), and input resistance (Fig. 4C). These relationships were not significant for primary motoneurons alone, which are clustered at the dorsal edge of the motor column, but were for the secondary motoneurons. There were also some developmental differences in the motoneurons between embryos and larvae. For primary motoneurons, which we could readily identify at both developmental stages, there was a statistically significant dorsal shift in their average position, a significant increase in soma size and a significant decrease in input resistance (Table 1).

Over all, these quantitative data suggest that the topographic map of recruitment for motoneurons in larvae is evident in embryos, with features like size and input resistance possibly contributing to the recruitment pattern. There was also a dorsal shift in the location of primary motoneuron somata during development that we show later in this paper is likely associated with the addition of neurons ventrally. We next investigated the develop- ment of recruitment patterns of excitatory premotor interneurons that could be driving the developmental changes in motoneuron firing described earlier.

\section{Firing of premotor excitatory interneurons during fictive swimming in prehatching embryos}

The CiD cells are premotor excitatory interneurons that are active during escape and fast swimming movements in zebrafish larvae (Ritter et al., 2001; Bhatt et al., 2007; McLean et al., 2007). Therefore, they may be very important in shaping motor activity during escape and fast swimming movements. In support of this, preliminary work ablating CiDs has demonstrated a detrimental effect on escapes (Fan and Hale, 2005), but none on slower swimming (McLean et al., 2007). A recent study recorded $\mathrm{CiD}$ neuron activity during swimming in zebrafish embryos, using a transgenic line of fish in which CiDs are selectively labeled using the transcription factor alx (Kimura et al., 2006). They found that CiDs could be categorized into more dorsal "strong-movement" types and more ventral "weakmovement" types. Here, we have used this same line of fish to take a closer look at the relationship between recruitment and position in embryos, to see whether there is also a continuous dorsoventral gradient as there is in larvae (McLean et al., 2007).

Figure 5 illustrates recordings from CiDs in 2-d-old embryos, combined with nerve recordings to monitor motor output. Consistent with the study by Kimura and colleagues, we found that in embryos the most dorsal CiDs, which are displaced from the more ventral, main CiD population (displaced CiDs), were only active during the initial burst of motor activity following a stimulus and not during subsequent cycles of swimming (Fig. $5 A$ ). Specifically, at no time during 113 episodes from 15 different embryos did displaced CiDs fire cyclically during swimming, even after suprathreshold stimuli (Fig. 5A, gray trace inset). The first motor burst represents the initial escape bend, which prompted their designation as strong-movement class (Kimura et al., 2006). It is worth noting, however, that by larval stages the dorsally displaced CiD cells not only fire during the initial burst, but also cyclically at very high frequencies of swimming (McLean et al., 2008). Thus, there appears to be a change in the contribution of these distinctive cells to motor behaviors with age.

Cells targeted at mid-dorsal regions in alx:GFP fish had a different morphology from dorsally displaced CiDs. As shown in Figure $5 B$, CiDs in this region had an ascending branch of the axon that extended at least one body segment. CiD interneurons were originally characterized anatomically by a major descending axon and minor ascending one (Bernhardt et al., 1990; Hale et al., 2001), so these could be considered CiDs with a "classic" morphology. Cells targeted even more ventrally in alx:GFP fish also had slightly different morphologies from dorsally displaced CiDs and classic CiDs. In embryos, these ventral-most CiDs typically had a shorter ascending branch and very often multiple branch points coming of the main descending axon (Fig. $5 C$, white arrow in gray inset). This may reflect a newly growing axon and thus be indicative of their relative youth.

With respect to developmental changes in firing (excluding the dorsally displaced CiDs which do not fire cyclically during swimming), there was a significant increase from embryos to 


\section{A 2 day old displaced $\mathrm{CiD}$ interneuron}

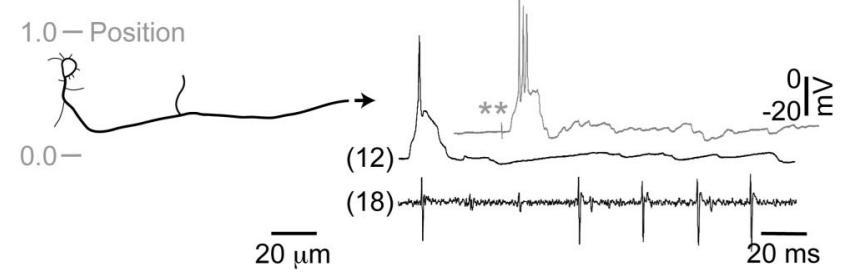

B 2 day old CiD interneuron (dorsal)

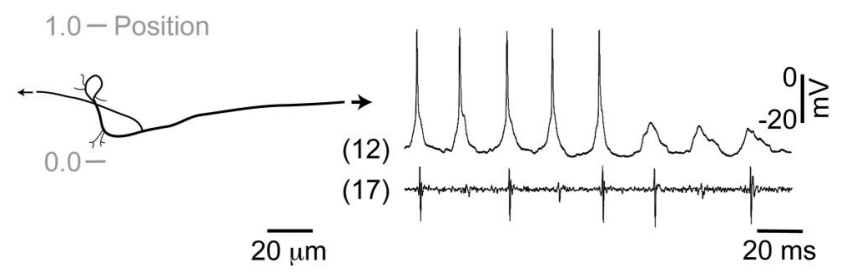

C 2 day old $\mathrm{CiD}$ interneuron (ventral)
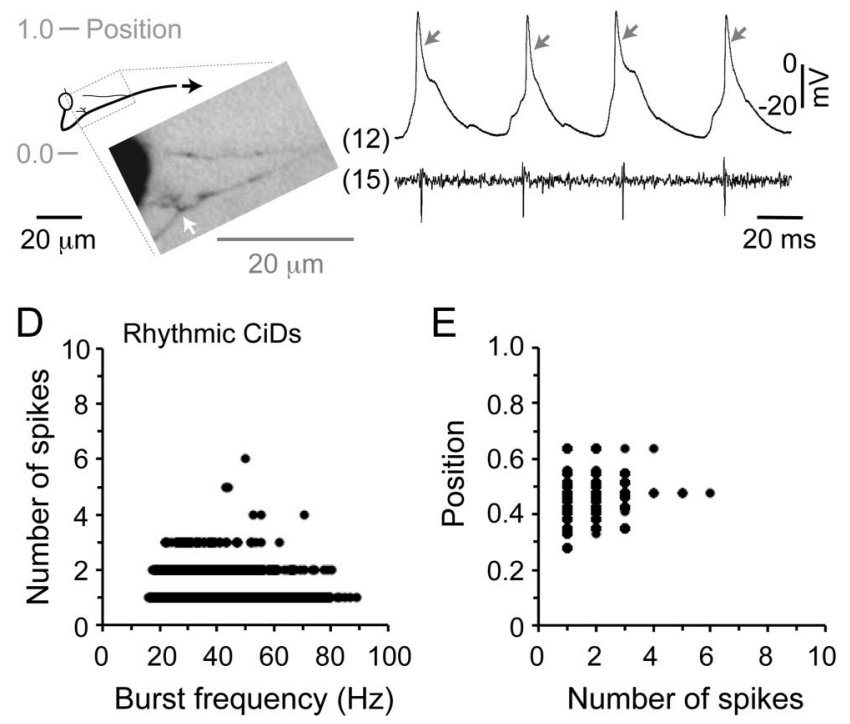

Figure 5. Excitatory interneuron firing during fictive swimming. $A$, Recording from a dorsally displaced CiD interneuron in an embryo, with the reconstructed cells on the left and the corresponding physiology immediately to the right. Gray-shaded lines indicate the position with respect to the dorsal (1.0) and ventral $(0.0)$ edges of spinal cord. The arrow indicates continuation of the axon out of the field of view. The respective segment from which the cell and the nerve recording were obtained is indicated in parentheses. The gray-shaded trace inset is from the same CiD cell and illustrates only the patch recording in response to a suprathreshold stimulus (double asterisk). While the stronger stimulus could not elicit firing in consecutive cycles, it did, however, cause more spikes. $\boldsymbol{B}$, Recording from a dorsally located CiD interneuron is organized as detailed for $\boldsymbol{A}$. Note the prominent ascending axon. $\boldsymbol{C}$, Recording from a ventrally located CiD interneuron is organized as detailed for $\boldsymbol{A}$. A photograph detailing the boxed region of the cell is inset, and a white arrow points out the growth of what is most likely new axon. Gray arrows point out the relatively broad action potentials of this cell. $\boldsymbol{D}$, Plot of the number of spikes per cycle generated by excitatory interneurons versus swimming frequency. Data represent 12,641 cycles from 20 CiD cells (black circles). $\boldsymbol{E}$, Plot of the somatic dorsoventral position of excitatory interneurons versus the number of spikes. Graph is plotted from the same dataset in $\boldsymbol{D}$.

larvae in the extent of firing per cycle for rhythmically active CiDs throughout the dorsoventral extent of spinal cord (Table 1). Like motoneurons, the most noticeable variability in spiking per cycle was at lower swimming frequencies in embryos (Fig. 5D). The same pattern is also seen in larvae, although there is a much greater degree of variability at low frequencies (McLean et al., 2008). Also like motoneurons, there was no clear relationship between the capacity to fire multiply and the dorsoventral location of the CiD cell (Fig. 5E).

These recordings demonstrate that some CiDs in embryos are active even at the lowest frequencies of swimming, with members of the population involved over the whole range of swimming frequencies. In larvae, these cells are recruited at higher frequencies of swimming and their ablation has no effect on low-frequency swimming (McLean et al., 2007, 2008). The CiD contributions to the entire frequency range of embryonic swimming is consistent with the observation that there is larger bending at both head and tail at all frequencies of embryonic swimming, but this is seen only at higher frequencies in larval fish.

These data also provide evidence for changes in the premotor excitatory drive during development. For instance, the most dorsally displaced cells fire only during the initial escape burst in embryos, but in larvae they also fire during very fast swimming (McLean et al., 2008). In addition, CiDs that fire one or two action potentials per swim cycle in embryos begin to fire multiply per cycle in larvae. Finally, the ventral-most CiDs appear to still be developing based on the presence of ascending axons sprouting from the main descending one. So, these data are consistent with changes in the flexibility of the motor pattern in the freeswimming larvae resulting from reconfigurations in pre-existing dorsal excitatory circuitry and the possible addition of new ventral excitatory circuitry.

\section{Measuring the position, recruitment, size, and input resistance of CiDs in embryos}

In Figure 6, we compare the same features of recruitment for rhythmically active CiDs in embryos, as we did for motoneurons. We did not include the dorsally displaced CiDs in this dataset, since they are not active during swimming in embryos. As in our prior studies of larvae (McLean et al., 2007), there were significant relationships between dorsoventral position and both the minimum frequency of activation during swimming (Fig. 6A) and input resistance (Fig. 6C), but not soma size (Fig. 6B).

Because we could identify dorsally displaced CiDs in both embryos and larvae, we compared their average position. There was a significant dorsal shift in soma location during development like primary motoneurons (Table 1 ). There was a significant change (decrease) in soma size, but not in input resistance (Table 1). In our original report relating recruitment order to soma size and input resistance in larvae, we were unable to target these cells (McLean et al., 2007), but we could in a subsequent study using the alx:GFP transgenic line in which they are labeled. There we found they were recruited only at the highest swimming frequencies in accord with their extreme dorsal position (McLean et al., 2008). From these later recordings, we also found that the dorsally displaced CiDs have higher input resistances than we would predict based on their location and order of recruitment (Table 1). This, along with their displaced location and changes in activity during development, represents several specialized features of these cells.

In summary, the topographic pattern of recruitment of both motoneurons and $\mathrm{CiD}$ interneurons first described in freeswimming larvae is also evident before hatching in embryos. The dorsally displaced cells in embryos do not participate in rhythmic swimming as they do in larvae, but rather are active only at the beginning of the episode, during what is the initial escape bend. Surprisingly, in both embryos and larvae, these displaced CiDs have higher input resistances than would be predicted based on their participation in the fastest, most powerful motor behaviors (i.e., escapes and fast swimming). Thus, at either stage, the 


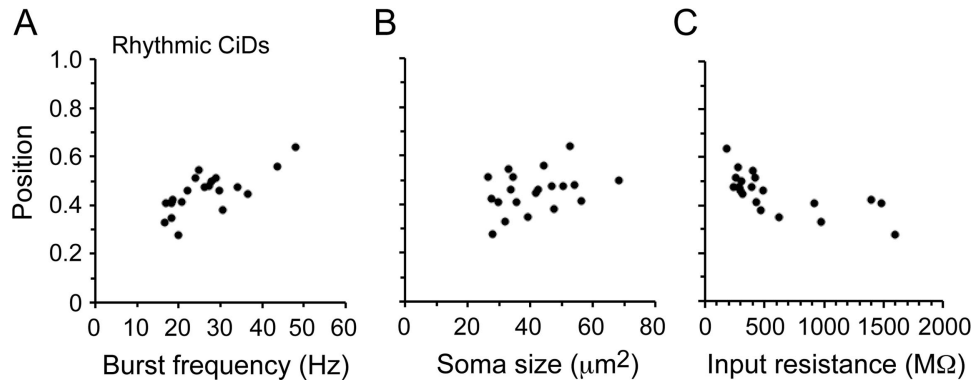

Figure 6. Excitatory interneuron recruitment, size, and excitability in embryos. A, Plot of spinal cord position versus swimming frequency for 20 CiD somata (open black circles). Correlation values: $r=0.72, p<0.001, n=20 . \boldsymbol{B}$, Plot of soma position versus soma size for the same CiDs in $\boldsymbol{A}$. Correlation values: $r=0.34, p=0.15, n=20$. C, Plot of soma position versus input resistance for the same CiDs in $\boldsymbol{A}$ and $\boldsymbol{B}$. Correlation values: $r=-0.68, p<0.001, n=20$. Each data point in $\boldsymbol{A}-\boldsymbol{C}$ represents the mean value for each cell.

best predictor of recruitment is the location of the neurons, which, as we show below, is also related to their time of differentiation.

\section{In vivo imaging of CiD differentiation using the photoconvertible protein Kaede}

In the prior study examining the development and activity of CiDs mentioned above, the cells were divided into two groups, a dorsally located strong-movement class and a ventrally located weakmovement class (Kimura et al., 2006). By expressing the green color change protein Kaede selectively in CiDs, Kimura and colleagues could flash entire fish with UV light and turn red those cells that were present at that age and expressing Kaede. Using this in vivo test of neuronal differentiation, they demonstrated that the strong-movement-class cells were earliest born.

Our data raise the possibility that rather than just two functional groups based upon age, there might be a progressive addition of $\mathrm{CiD}$ interneurons to the bottom of spinal cord in conjunction with the development of increasingly more refined swimming movements. To test this idea, we used the same alx: Kaede line and instead systematically converted fish at $1 \mathrm{~d}$ intervals and raised them to larval stages, to ask where the earlier differentiating red neurons were relative to later differentiating green ones.

Figure 7 illustrates the results of these experiments designed to assess the order of differentiation of CiD cells that occupy different places in the topographic map of recruitment in larval fish. Consistent with studies in embryos (Kimura et al., 2006), photoconversion of 1-d-old alx:Kaede fish labeled only the dorsal-most displaced CiD cells with red protein when examined in 4-d-old fish (Fig. 7A), indicating that these neurons were the first to differentiate. Green protein was still produced in these dorsal cells and in the numerous more ventral cells that must have differentiated after the flash (Fig. $7 \mathrm{~B}, \mathrm{C}$ ). When viewed in cross section, it was clear that the older CiD cells occupied more dorsal and lateral positions than newer CiD cells (Fig. 7D).

We next systematically examined the dorsoventral patterning of $\mathrm{CiD}$ differentiation. In Figure $7 E$, we have plotted the dorsoventral distribution of every alx:Kaede cell examined (red or green). The greatest concentration of alx:Kaede-labeled CiD cells in larvae was at the dorsoventral level of 0.5-0.6, with numbers falling dorsally and ventrally from there. If there was a progressive addition of cells ventrally, then converting alx:Kaede protein at successive $1 \mathrm{~d}$ intervals should reveal an increasing proportion of older cells (any red protein) in more dorsal positions at the expense of younger cells (pure green protein). This is, in fact, what we observed. Cells that had differentiated by the first day were located most dorsally (Fig. $7 F$ ). Cells that had differentiated by the second day (including all before then) were located in mid-level to dorsal regions (Fig. $7 G$ ). Cells that had differentiated by the third day (including all before then) were located in more ventral to dorsal regions (Fig. $7 H$ ). In each case, the pure green, youngest cells always occupied the ventral-most regions, when compared with older cells. This very regular pattern is consistent with the idea that the dorsoventral position of cells is a reflection of when they differentiated. This dorsoventral patterning of development coupled with the dorsoventral pattern of recruitment argues strongly for the systematic emergence of spinal circuitry according to its participation in different swimming frequencies in larvae.

\section{In vivo imaging of MCoD differentiation using Kaede}

To test this idea further, we examined the time course of differentiation of another class of premotor excitatory interneuron, MCoD interneurons (Hale et al., 2001; Higashijima et al., 2004a). These cells are known to participate in the slowest speeds of larval locomotion (Ritter et al., 2001; McLean et al., 2007) and their ablation disrupts the normal expression of slow swimming movements (McLean et al., 2007). MCoDs are located relatively ventrally and, like motoneurons and CiD interneurons, display the greatest variability in spiking at lower swimming frequencies in larvae (McLean et al., 2008).

If a ventral location and a contribution to slower swimming are characteristics of youth, we would predict that MCoDs differentiate very late in conjunction with the later development of the lowest frequency larval movements. MCoDs are not labeled by the transcription factor alx, so we used another transgenic line of fish that labels all neurons with Kaede using the neural-specific promoter, $\mathrm{HuC}$. The MCoDs have an unusually lateral location that makes them easily visible in the transgenic line (McLean et al., 2008). As shown in Figure 8, MCoDs are located ventrally (Fig. $8 A$ ), have a commissural axonal process (Fig. 8D) and are labeled by $H u C$ :Kaede at larval stages of development (Fig. $8 B, C)$. In Figure $8 E$, we plotted the dorsoventral position of every MCoD labeled with $H u C$ :Kaede (red or green). Although MCoDs occupy a distinctive location in the ventrolateral neuropil, we also positively identified them using a backfilling approach (Hale et al., 2001; Ritter et al., 2001). Using the same approach as with alx:Kaede fish, we found that $\mathrm{MCoDs}$ were not expressing the $\mathrm{HuC}$ promoter-driven transgene (which is an early expressed marker of neuronal identity) by $1 \mathrm{~d}$ of age (Fig. $8 \mathrm{~F}$ ) or by $2 \mathrm{~d}$ of age (Fig. $8 G$ ) but had started to differentiate by $3 \mathrm{~d}$ of age (Fig. $8 H$ ). This is well after most of the CiD neurons have differentiated, based on the alx marker, which is expressed later than $H u C$. As with CiD cells, the dorsal-most MCoDs appeared to be the oldest MCoDs (Fig. $8 H$ ). These data confirm that the MCoDs, which are very ventral and participate in the lowest frequency swimming movements of larval fish, do not begin to appear until later in development when the lowest frequency, predominantly tail-based movements of larvae become apparent. Thus, neurons develop in an orderly way from those driving the high-frequency movements in larvae to those driving low- 

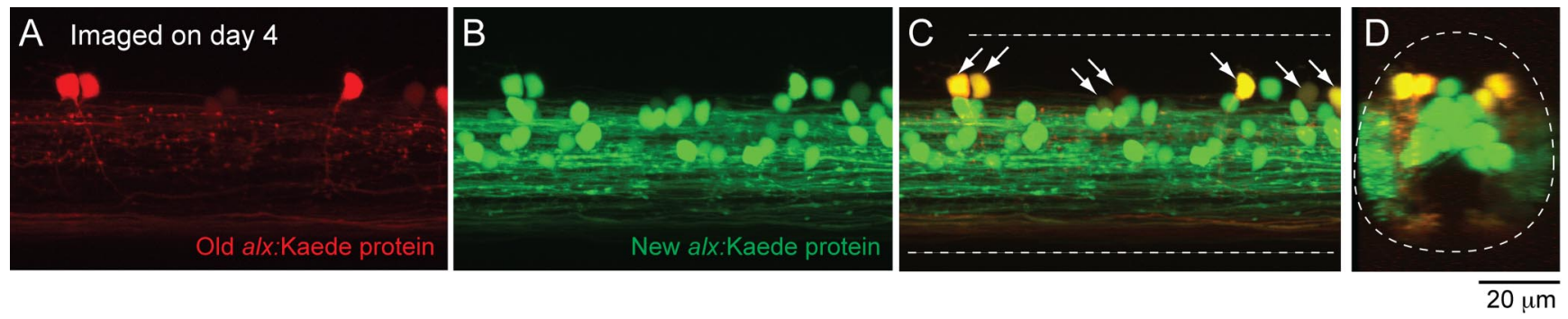

$\mathrm{E}$

alx:Kaede-labeled cells (4 days old)

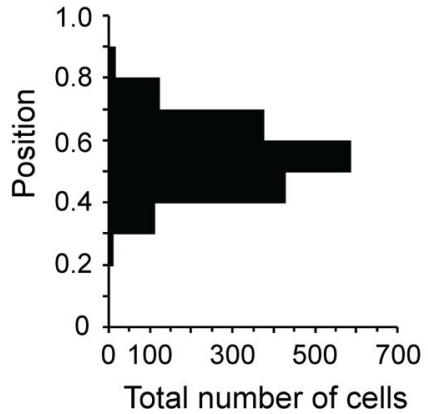

F Converted at 24-30 hpf (Imaged on day 4)

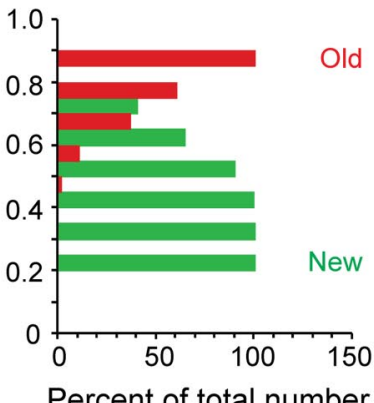

G Converted at $48-54 \mathrm{hpf}$ (Imaged on day 4)

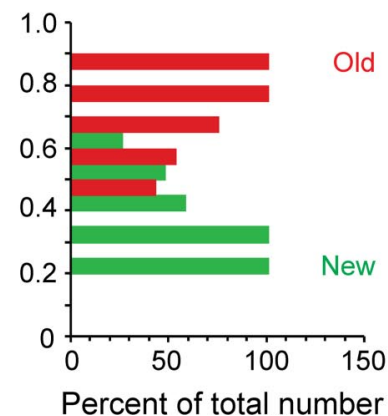

H Converted at 72-78 hpf (Imaged on day 4)

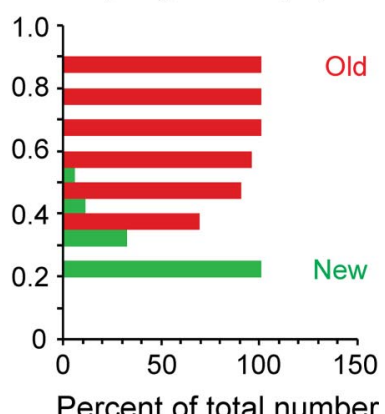

Figure 7. Kaede labeling to track alx-labeled CiD interneuron differentiation in vivo. A-C, Confocal $z$ stack (54 $\mu \mathrm{m}$ in depth) from midbody (segment 15) illustrates alx cells labeled with the photoconvertible protein Kaede in a 4-d-old larva. Cells expressing Kaede at the time of photoconversion ( $24-30 \mathrm{hpf}$ or $1 \mathrm{~d} \mathrm{old}$ ) contain red labeling at day $4(\boldsymbol{A})$, while those that differentiated after day 1 have only green labeling $(\boldsymbol{B})$. A merged image demonstrates that older, red cells continued to express new, green Kaede protein and so are yellow (at arrows) (C). D, An optical cross section of the z stack in $\boldsymbol{A}$-C illustrates that older cells are more dorsally and laterally located. $\boldsymbol{E}$, A bar chart illustrating the dorsoventral distribution of all 1620 alx:Kaede-labeled cells from 21 larvae. $\boldsymbol{F}$ - $\boldsymbol{H}$, Bar charts illustrating the percentage of alx:Kaede-labeled cells imaged on day 4 at different dorsoventral locations that contained any red Kaede protein (old, red bars) or only green Kaede protein (new, green bars) when converted at either $24-30 \mathrm{hpf}$ (day 1, F), $48-54 \mathrm{hpf}$ (day 2, G), or $72-78 \mathrm{hpf}($ day $3, \boldsymbol{H}$ ).

frequency ones, both within CiDs and across the CiD and the MCoD interneuron populations.

\section{Discussion}

The main conclusion of this work is that the topographic map we described for larval fish (McLean et al., 2007), which relates the dorsoventral location of a neuron to the swimming frequency and speed at which it is recruited, develops via an orderly addition of the neurons driving different frequencies of movement in the larvae. The dorsal-most excitatory neurons that ultimately drive the highest frequency movements come first and increasingly slower ones are layered on ventrally during development. Because the speed of movement through the water increases with bending frequency, the pattern relates the order of differentiation to both the bending frequency and the speed of swimming (Müller and van Leeuwen, 2004). Thus, our findings tie the larval topographic recruitment pattern to a systematic pattern of network differentiation in which larval networks develop in order from fast to slow.

Multiple, converging lines of evidence support this conclusion. First, early embryonic swimming at any frequency looks like fast larval swimming, with substantial lateral movements of both the head and tail. This is the case even though the range of burst/ bending frequencies in embryos overlaps that in larvae. The more localized larval swimming movements, involving tail displacement with little head movement, are not evident in early embryos, but occur during the slowest movements in larvae. Evidently the neurons driving the whole range of frequencies in the developing embryo end up driving just the upper range of frequencies in the larvae. This might be a consequence of changes in cellular or network properties as the embryonic neurons continue to differentiate into the larval stage. A second line of evidence that the networks driving fast movements in larvae develop first is that the motor pattern in embryos more closely resembles the faster, higher frequency swimming motor pattern in larvae. It shows less variable, shorter burst durations and a lack of a regular pattern of scaling of intersegmental delay, consistent with an absence of neurons capable of driving a range of burst durations and consistent intersegmental motor patterns. Variable burst durations and scaled intersegmental delays develop later in conjunction with the addition of new excitatory premotor interneurons that drive slower larval swimming. Third, patch recordings and the morphology of spinal neurons also support an ordered pattern of differentiation. In embryos, alx-labeled interneurons include extremely dorsally displaced CiDs, CiDs with a prominent ascending axon, and ventral CiDs that look like they are in the process of growing an ascending axon. This suggests that the ventral-most CiDs in embryos differentiate into CiDs with a classic morphology (which participate in fast larval swimming) and are displaced dorsally by newly emerging CiDs in larvae, which now occupy the ventral-most locations. A general dorsal-ward shift is supported by measurements of soma location of identifiable classes of patched cells during development (see Table 1). Finally, the most direct piece of evidence for dorsoventral patterning during the differentiation of excitatory interneurons is provided by the Kaede experiments. The data from CiDs show that they are arranged with the first to differentiate located most dorsal in larvae and increasingly younger neurons stacked below them. The MCoDs, which are active only at the slowest speeds (McLean et al., 2008), differentiate very late and are located most ventrally, showing that the developmental order, like the recruitment pattern and topographic map of swimming frequency/ speed, crosses cell types.

Collectively, these data reveal an organization in which the excitatory premotor interneurons and motoneurons responsible 

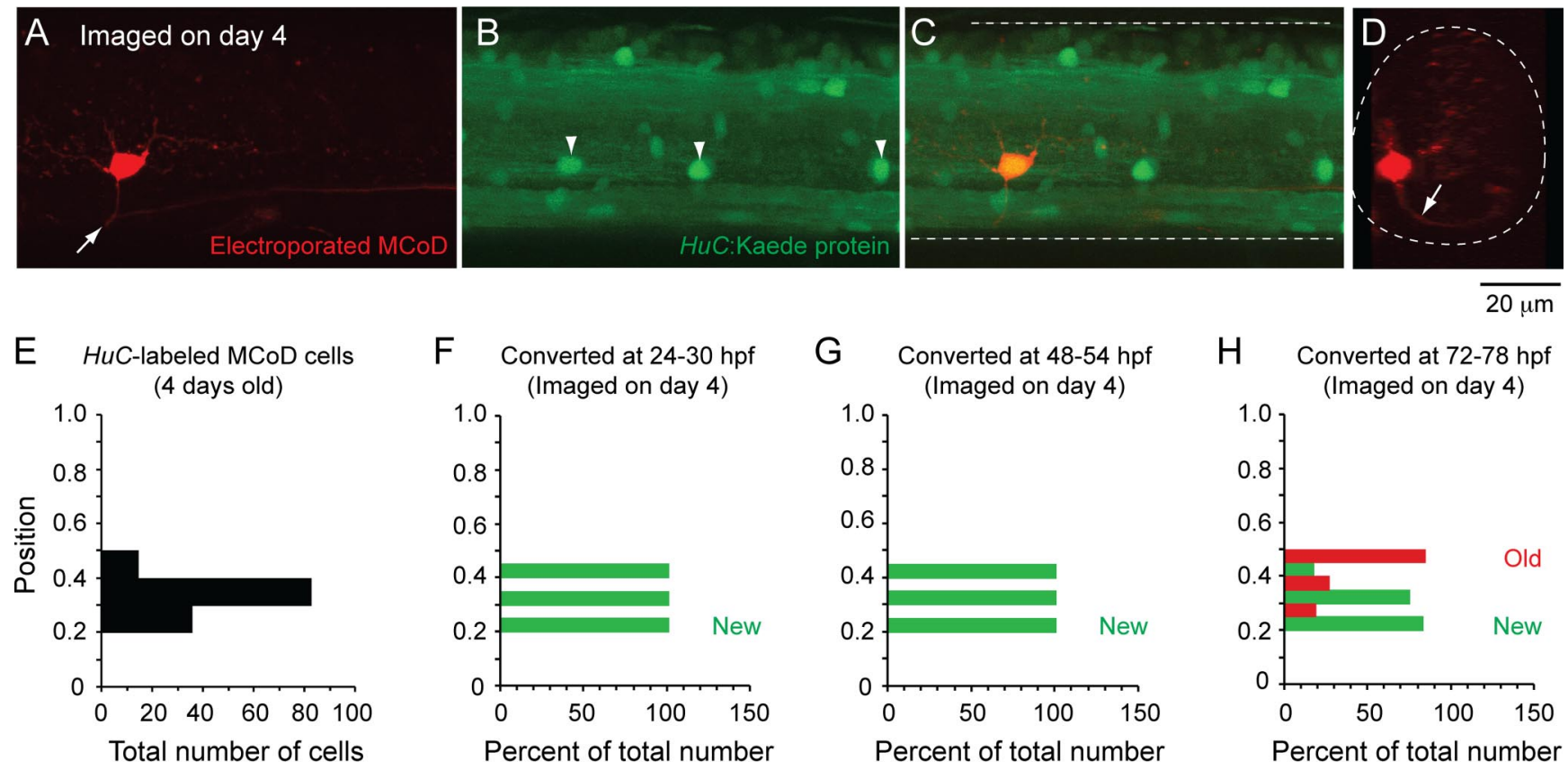

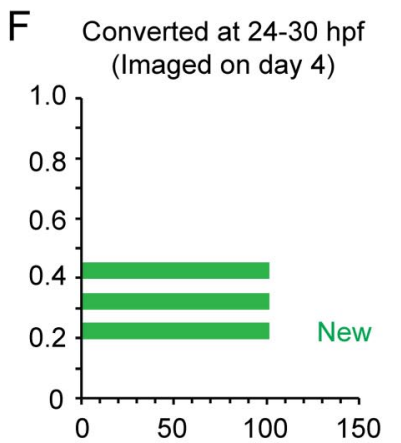

Percent of total number
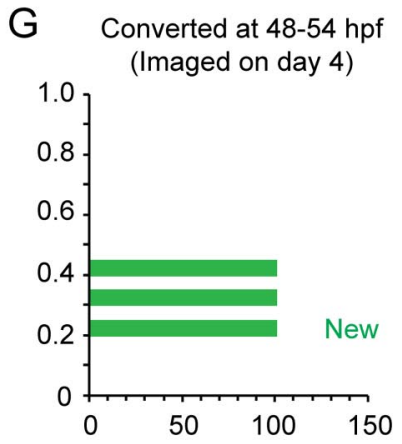

Percent of total number
$\mathrm{H} \quad$ Converted at $72-78 \mathrm{hpf}$ (Imaged on day 4)

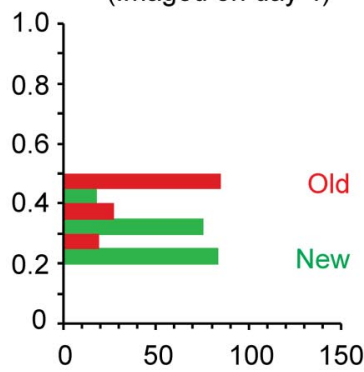

Percent of total number

Figure 8. Kaede labeling to track MCoD differentiation in vivo. A-C, Confocal z stack (47 $\mu \mathrm{m}$ in depth) from midbody (segment 15) illustrates an MCoD electroporated with dye in a 4-d-old transgenic HuC:Kaede fish. $\boldsymbol{A}$, The MCoD axon crosses spinal cord at the white arrow. $\boldsymbol{B}$, White arrowheads mark laterally located cells, which are likely MCoDs. $\boldsymbol{C}$, Merged image demonstrates one of the laterally located $H u C$ :Kaede cells is indeed an MCoD and so is yellow. $D$, An optical cross section of the $z$ stack in $A$ illustrates that the MCoD is located ventrally and has a commissural process (at white arrow). $\boldsymbol{E}$, A bar chart illustrating the dorsoventral distribution of all 131 HuC:Kaede-labeled MCoD cells from 32 4-d-old larvae. $\boldsymbol{F}$ - $\boldsymbol{H}$, Bar charts illustrating the percentage of HuC:Kaedelabeled MCOD cells imaged on day 4 at different dorsoventral locations that contained any red Kaede protein (old, red bars) or only green Kaede protein (new, green bars) when converted at either 24-30 hpf (day 1, F), $48-54$ hpf (day 2, G), or 72-78 hpf (day 3, $\boldsymbol{H})$.

for fast escape and swimming movements in posthatching, freeswimming larvae develop first and occupy the dorsal-most positions. Those neurons driving increasingly slower larval movements are layered on below during development. The result is the topographic pattern of recruitment in free-swimming larvae.

In retrospect, there were pieces of evidence available for many years, including some of our own work, that suggested such a pattern, but we and others did not realize it represented a systematic pattern of organization within and across cell types in the spinal cord. From the earliest studies of zebrafish it was clear that the oldest, primary motoneurons (actually named so based on their being born first) were most dorsal and were active in fast movements, whereas the more ventral secondary ones were involved in slower movements (Myers et al., 1986; Westerfield et al., 1986; Liu and Westerfield, 1988). Whether there was also a systematic topographic order with respect to function and developmental age within secondaries was unknown. Among interneurons, there was evidence that dorsal CiD interneurons were activated during fast escape movements and ventral MCoDs during slow swimming movements in larvae (Ritter et al., 2001), but the presence of an overall age and functional topography across these cell classes was not recognized. The strongest prior evidence for a possible age, function, and position relationship came from studies in zebrafish embryos using Kaede in the alx transgenic line we used, which showed that a group of strong-movement cells were generally dorsal and differentiated first and a weakmovement group came later and were more ventral (Kimura et al., 2006). Our data show that this represents a piece of a systematic pattern that develops in the embryo, extends across both motoneurons and interneurons, and leads to a topographic relationship between dorsoventral position and recruitment in the free-swimming larva after hatching when a broad swimming repertoire is evident.
Is this pattern unique to zebrafish or is there any evidence that this may be a general pattern of organization for spinal motor systems? There are striking parallels between the pattern of motor behavioral development in zebrafish and that of Xenopus laevis tadpoles, one of the most important models for studying spinal CPGs (Roberts et al., 1998; McLean et al., 2000; Sillar et al., 2008). At stage 37/38 (Nieuwkoop and Faber, 1994), just before hatching, Xenopus embryos behave very much like 2-d-old zebrafish: they are sedentary but produce ballistic swimming movements when startled (Roberts et al., 1981). By stage 42 ( $\sim 24$ h later at $\left.23^{\circ} \mathrm{C}\right)$, Xenopus larvae are still largely quiescent, but the motor pattern has transformed from one with brief motor bursts and fixed intersegmental delays into one with variable motor bursts and properly scaled delays (Sillar et al., 1991; Tunstall and Sillar, 1993), just as is seen at later stages in zebrafish. Later still, Xenopus swim slowly using more refined movements at the tail, also as in zebrafish (von Seckendorff-Hoff and Wassersug, 1986). The pattern of changes in firing from single to multiple spikes we observed during the development of zebrafish swimming was first described in Xenopus and most likely contributes to similar changes in their motor patterns (Sillar et al., 1992; Zhang et al., 2009). The recent description of dorsoventral gradients in excitability and connectivity in Xenopus spinal cord suggests that a similar dorsoventral pattern of function could be present in developing frogs (Li et al., 2007b; Pineda and Ribera, 2008). These similarities argue for the general applicability of our findings to developing swimming vertebrates, including a tetrapod (Xenopus).

Is there a similar pattern in other tetrapods? There is a substantial body of evidence that suggests that the pattern of motor development of tetrapods, including humans, has striking parallels with that in fishes and frogs, raising the possibility of similar underlying patterns of circuit development (Hamburger et al., 1965; Narayanan et al., 1971; Bekoff and Trainer, 1979; de Vries 
et al., 1982; Jacobson and Hollyday, 1982; Landmesser and O’Donovan, 1984; Bradley and Bekoff, 1990; Cazalets et al., 1990; Westerga and Gramsbergen, 1990; Suzue and Shinoda, 1999). To give just one example from an extensive literature, studies of the development of movement in utero in humans show that the first movements are startle responses, followed by broad movements of the body and only later by more localized movements of the limbs (de Vries et al., 1982). The parallels to the developmental order in zebrafish are striking (de Vries et al., 1982, their Fig. 9).

Although not much is known about the order of differentiation of spinal neurons driving different movement speeds or amplitudes in other vertebrates, evidence from a variety of systems points to the possibility that the early development of large neurons that are hard to excite, but that have powerful effects, may be widespread in the nervous system of both tetrapods and fishes. The hindbrain reticulospinal neurons driving escape responses in the fish are large with high thresholds and low input resistances and differentiate very early (Mendelson, 1985; Faber et al., 1989). Startle neurons in the pontine reticular formation of mammals are similarly large and of high threshold and also differentiate early (Altman and Bayer, 1980; Lingenhöhl and Friauf, 1994).

This developmental order may extend beyond motor systems as well, given that the largest neurons differentiate first in many regions of the brain including pyramidal neurons in cortical layers 5 and 6 (Angevine and Sidman, 1961), Purkinje neurons in the cerebellum (Hatten and Heintz, 1995), and the magnocellular neurons in the thalamus (Rakic, 1977). In thalamus, the magnocellular system deals with fast coarse visual processing in contrast to the more refined analysis of the smaller parvocellular neurons (Livingstone and Hubel, 1988).

While these are only bits of evidence, they suggest that a careful analysis might reveal that a pattern of early emergence of high-threshold, strong, often larger neurons involved in gross movements or strong synaptic effects is widespread throughout the nervous system, including the brain. This could reflect the evolutionary origin of the brain from the spinal cord, where there may have been a simple, primitive developmental sequence with high-threshold, fast neurons that drive gross movements developing first, followed by later differentiation of lower-threshold neurons driving more refined movements. The orderly development of the topographic map of function that we describe may therefore represent a primitive patterning that still underlies features of the organization of the nervous system throughout vertebrates.

\section{References}

Altman J, Bayer SA (1980) Development of the brain stem in the rat. IV. Thymidine-radiographic study of the time of origin of neurons in the pontine region. J Comp Neurol 194:905-929.

Angevine JB Jr, Sidman RL (1961) Autoradiographic study of cell migration during histogenesis of cerebral cortex in the mouse. Nature 192:766-768.

Beattie CE, Hatta K, Halpern ME, Liu H, Eisen JS, Kimmel CB (1997) Temporal separation in the specification of primary and secondary motoneurons in zebrafish. Dev Biol 187:171-182.

Bekoff A, Trainer W (1979) The development of interlimb co-ordination during swimming in postnatal rats. J Exp Biol 83:1-11.

Berkowitz A (2008) Physiology and morphology of shared and specialized spinal interneurons for locomotion and scratching. J Neurophysiol 99:2887-2901.

Bernhardt RR, Chitnis AB, Lindamer L, Kuwada JY (1990) Identification of spinal neurons in the embryonic and larval zebrafish. J Comp Neurol 302:603-616.

Bhatt DH, Otto SJ, Depoister B, Fetcho JR (2004) Cyclic AMP-induced repair of zebrafish spinal circuits. Science 305:254-258.

Bhatt DH, McLean DL, Hale ME, Fetcho JR (2007) Grading movement strength by changes in firing intensity versus recruitment of spinal interneurons. Neuron 53:91-102.

Bradley NS, Bekoff A (1990) Development of coordinated movement in chicks: I. Temporal analysis of hindlimb muscle synergies at embryonic days 9 and 10. Dev Psychobiol 23:763-782.

Budick SA, O'Malley DM (2000) Locomotor repertoire of the larval zebrafish: swimming, turning and prey capture. J Exp Biol 203:2565-2579.

Buss RR, Drapeau P (2001) Synaptic drive to motoneurons during fictive swimming in the developing zebrafish. J Neurophysiol 86:197-210.

Cazalets JR, Menard I, Crémieux J, Clarac F (1990) Variability as a characteristic of immature motor systems: an electromyographic study of swimming in the newborn rat. Behav Brain Res 40:215-225.

Crone SA, Zhong G, Harris-Warrick R, Sharma K (2009) In mice lacking V2a interneurons, gait depends on speed of locomotion. J Neurosci 29:7098-7109.

de Vries JI, Visser GH, Prechtl HF (1982) The emergence of fetal behaviour. I. Qualitative aspects. Early Hum Dev 7:301-322.

Downes GB, Granato M (2006) Supraspinal input is dispensable to generate glycine-mediated locomotive behaviors in the zebrafish embryo. J Neurobiol 66:437-451.

Drapeau P, Ali DW, Buss RR, Saint-Amant L (1999) In vivo recording from identifiable neurons of the locomotor network in the developing zebrafish. J Neurosci Methods 88:1-13.

Faber DS, Fetcho JR, Korn H (1989) Neuronal networks underlying the escape response in goldfish. General implications for motor control. Ann N Y Acad Sci 563:11-33.

Falgairolle M, de Seze M, Juvin L, Morin D, Cazalets JR (2006) Coordinated network functioning in the spinal cord: an evolutionary perspective. J Physiol Paris 100:304-316.

Fan J, Hale ME (2005) Excitatory descending spinal interneurons influence the degree of axial bending during startles of larval zebrafish. Soc Neurosci Abstr 31:751.14.

Goulding M (2009) Circuits controlling vertebrate locomotion: moving in a new direction. Nat Rev Neurosci 10:507-518.

Grillner S (2006) Biological pattern generation: the cellular and computational logic of networks in motion. Neuron 52:751-766.

Grillner S, Kashin S (1976) On the generation and performance of swimming in fish. In: Neural control of locomotion (Herman RM, Grillner S, Stein PSG, Stuart DG, eds), pp 181-201. New York: Plenum.

Hale ME, Ritter DA, Fetcho JR (2001) A confocal study of spinal interneurons in living larval zebrafish. J Comp Neurol 437:1-16.

Hamburger V, Balaban M, Oppenheim R, Wenger E (1965) Periodic motility of normal and spinal chick embryos between 8 and 17 days of incubation. J Exp Zool 159:1-13.

Hatten ME, Heintz N (1995) Mechanisms of neural patterning and specification in the developing cerebellum. Annu Rev Neurosci 18:385-408.

Higashijima S, Schaefer M, Fetcho JR (2004a) Neurotransmitter properties of spinal interneurons in embryonic and larval zebrafish. J Comp Neurol 480:19-37.

Higashijima S, Masino MA, Mandel G, Fetcho JR (2004b) Engrailed-1 expression marks a primitive class of inhibitory spinal interneuron. J Neurosci 24:5827-5839.

Jacobson RD, Hollyday M (1982) A behavioral and electromyographic study of walking in the chick. J Neurophysiol 48:238-256.

Kiehn O (2006) Locomotor circuits in the mammalian spinal cord. Annu Rev Neurosci 29:279-306.

Kimura Y, Okamura Y, Higashijima S (2006) alx, a zebrafish homolog of Chx10, marks ipsilateral descending excitatory interneurons that participate in the regulation of spinal locomotor circuits. J Neurosci 26:5684-5697.

Landmesser LT, O’Donovan MJ (1984) Activation patterns of embryonic chick hind limb muscles recorded in ovo and in an isolated spinal cord preparation. J Physiol 347:189-204.

Li WC, Sautois B, Roberts A, Soffe SR (2007a) Reconfiguration of a vertebrate motor network: specific neuron recruitment and context-dependent synaptic plasticity. J Neurosci 27:12267-12276.

Li WC, Cooke T, Sautois B, Soffe SR, Borisyuk R, Roberts A (2007b) Axon and dendrite geography predict the specificity of synaptic connections in a functioning spinal cord network. Neural Dev 2:17.

Liao JC, Fetcho JR (2008) Shared versus specialized glycinergic spinal interneurons in axial motor circuits of larval zebrafish. J Neurosci 28:12982-12992. 
Lingenhöhl K, Friauf E (1994) Giant neurons in the rat reticular formation: a sensorimotor interface in the elementary acoustic startle circuit? J Neurosci 14:1176-1194.

Liu DW, Westerfield M (1988) Function of identified motoneurones and co-ordination of primary and secondary motor systems during zebra fish swimming. J Physiol 403:73-89.

Livingstone M, Hubel D (1988) Segregation of form, color, movement, and depth: anatomy, physiology, and perception. Science 240:740-749.

Masino MA, Fetcho JR (2005) Fictive swimming motor patterns in wild type and mutant larval zebrafish. J Neurophysiol 93:3177-3188.

McLean DL, Merrywest SD, Sillar KT (2000) The development of neuromodulatory systems and the maturation of motor patterns in amphibian tadpoles. Brain Res Bull 53:595-603.

McLean DL, Fan J, Higashijima S, Hale ME, Fetcho JR (2007) A topographic map of recruitment in spinal cord. Nature 446:71-75.

McLean DL, Masino MA, Koh IY, Lindquist WB, Fetcho JR (2008) Continuous shifts in the active set of spinal interneurons during changes in locomotor speed. Nat Neurosci 11:1419-1429.

Mendelson B (1985) Soma position is correlated with time of development in three types of identified reticulospinal neurons. Dev Biol 112:489-493.

Müller UK, van Leeuwen JL (2004) Swimming of larval zebrafish: ontogeny of body waves and implications for locomotory development. J Exp Biol 207:853-868.

Myers PZ, Eisen JS, Westerfield M (1986) Development and axonal outgrowth of identified motoneurons in the zebrafish. J Neurosci 6:2278-2289.

Narayanan CH, Fox MW, Hamburger V (1971) Prenatal development of spontaneous and evoked activity in the rat (Rattus norvegicus albinus). Behaviour 40:100-134.

Nieuwkoop PD, Faber J (1994) Normal table of Xenopus laevis (Daudin). New York: Garland.

Pineda RH, Ribera AB (2008) Dorsal-ventral gradient for neuronal plasticity in the embryonic spinal cord. J Neurosci 28:3824-3834.

Rakic P (1977) Genesis of the dorsal lateral geniculate nucleus in the rhesus monkey: site and time of origin, kinetics of proliferation, routes of migration and pattern of distribution of neurons. J Comp Neurol 176:23-52.

Ritter DA, Bhatt DH, Fetcho JR (2001) In vivo imaging of zebrafish reveals differences in the spinal networks for escape and swimming movements. J Neurosci 21:8956-8965.
Roberts A, Kahn JA, Soffe SR, Clarke JD (1981) Neural control of swimming in a vertebrate. Science 213:1032-1034.

Roberts A, Soffe SR, Wolf ES, Yoshida M, Zhao FY (1998) Central circuits controlling locomotion in young frog tadpoles. Ann N Y Acad Sci 860:19-34.

Saint-Amant L, Drapeau P (1998) Time course of the development of motor behaviors in the zebrafish embryo. J Neurobiol 37:622-632.

Sillar KT, Wedderburn JF, Simmers AJ (1991) The development of swimming rhythmicity in post-embryonic Xenopus laevis. Proc Biol Sci 246:147-153.

Sillar KT, Simmers AJ, Wedderburn JF (1992) The post-embryonic development of cell properties and synaptic drive underlying locomotor rhythm generation in Xenopus larvae. Proc Biol Sci 249:65-70.

Sillar KT, Combes D, Ramanathan S, Molinari M, Simmers J (2008) Neuromodulation and developmental plasticity in the locomotor system of anuran amphibians during metamorphosis. Brain Res Rev 57:94-102.

Stein PS, McCullough ML, Currie SN (1998) Spinal motor patterns in the turtle. Ann N Y Acad Sci 860:142-154.

Suzue T, Shinoda Y (1999) Highly reproducible spatiotemporal patterns of mammalian embryonic movements at the developmental stage of the earliest spontaneous motility. Eur J Neurosci 11:2697-2710.

Thorsen DH, Cassidy JJ, Hale ME (2004) Swimming of larval zebrafish: fin-axis coordination and implications for function and neural control. J Exp Biol 207:4175-4183.

Tunstall MJ, Sillar KT (1993) Physiological and developmental aspects of intersegmental coordination in Xenopus embryos and tadpoles. Sem Neurosci 5:29-40.

von Seckendorff-Hoff K, Wassersug RJ (1986) The kinematics of swimming in larvae of the clawed frog, Xenopus laevis. J Exp Biol 122:1-12.

Westerfield M, McMurray JV, Eisen JS (1986) Identified motoneurons and their innervation of axial muscles in the zebrafish. J Neurosci 6:22672277.

Westerga J, Gramsbergen A (1990) The development of locomotion in the rat. Brain Res Dev Brain Res 57:163-174.

Zhang HY, Li WC, Heitler WJ, Sillar KT (2009) Electrical coupling synchronises spinal motorneuron activity during swimming in hatchling Xenopus tadpoles. J Physiol 587:4455-4466. 